\title{
ALDH2, a novel protector against stroke?
}

\author{
Cell Research (2013) 23:874-875. doi:10.1038/cr.2013.76; published online 11 June 2013
}

In a recent paper published in Cell Research, an association between expression of mitochondrial aldehyde dehydrogenase (ALDH2), a mitochondrial chaperon expressed in the brain, and the prevalence of stroke is revealed. This finding indicates that ALDH2 may serve as a potential endogenous neuroprotective target and a promising therapeutic strategy for the management of stroke.

Stroke is one of the leading causes of death and a major reason of adult chronic disability as well as age-related cognitive decline and dementia [1]. Ischemic stroke represents $>80 \%$ of all stroke incidences with the remaining $20 \%$ due to primary hemorrhage. Proper management of the conventional risk factors for stroke, such as high blood pressure, elevated blood cholesterol, cigarette smoking, carotid stenosis, diabetes mellitus and heart failure, may reduce the incidence of stroke only to a certain degree, suggesting the existence of undiscovered or undefined risk factors $[1,2]$. The unidentified risk factors for stroke, in conjunction with unsatisfactory control of known risk factors (e.g., high cholesterol and hypertension), may explain the intimate clinical challenge or failure for stroke management. To this end, identification of novel risk factors may hold promises in the development of strategies for prevention and treatment of stroke. Ample evidence has implicated the importance of genetic predisposition in the onset and progression of stroke [2]. More recently, genome-wide association study (GWAS) approach has transformed the genetics of many complex chronic diseases and is just beginning to impact the field of stroke [3]. Genetic variants predisposing to ischemic stroke have been revealed by GWAS, such as two loci associated with atrial fibrillation (PITX2 and ZFHX3) linked to cardioembolic stroke and a locus on chromosome 9p21 tied to large-vessel stroke $[1,4,5]$. Nonetheless, the precise contribution of genetics to the etiology of stroke, in particular various stroke subtypes, remains somewhat elusive. Gene candidates that have been identified to be associated with stoke warrant further validation in a large independent data set to consolidate their causative role in the pathogenesis of stroke.

In a recent paper published in Cell Research, Guo and colleagues performed an unbiased proteomic examination and unveiled a unique role of deficiency in mitochondrial aldehyde dehydrogenase (ALDH2), the so-called "facial flash gene" responsible for detoxification of toxic aldehydes such as 4-hydroxy2-nonenal (4-HNE), in the pathogenesis of stroke [6]. In their study, overexpression or activation of ALDH2 conferred neuroprotection through clearance of 4-HNE whereas ALDH2 knockdown mitigated the neuroprotective property of PKCE. The PKCE-ALDH2 pathway was shown to mediate neuroprotection offered by moderate ethanol intake. Serum 4-HNE levels were inversely correlated with lifespan and elevated plasma 4-HNE levels were observed for at least 6 months following stroke onset. Perhaps the most intriguing evidence is that much higher initial plasma 4-HNE levels were associated with development of stroke in an 8-year follow-up study. These findings favor a likely role of ALDH2 in the prevalence of stroke or stroke-prone subjects, and furthermore, its therapeutic potential as a target in the management of stroke (Figure 1).

$A L D H 2$ is a human gene found on chromosome 12. All Caucasians are homozygous for $A L D H 2$ while approximately $50 \%$ of Asians are heterozygous and possess only one normal copy of the $A L D H 2$ gene and one mutant copy encoding an inactive mitochondrial isozyme [7]. A recent meta-analysis of GWAS identified a tight association between $A L D H 2$ genetic mutation and elevated blood pressure, a known risk factor for stroke, in Asian decedents [8]. This is supported by the recent observation favoring a crucial role for ALDH2 in the regulation of cardiovascular homeostasis in diabetes, alcoholism, endoplasmic reticulum stress, arrhythmias and ischemia-reperfusion injury [9-11]. Stroke is known to interrupt mitochondrial function and promote mitochondrial swelling and depolarization, leading to ultimate neuronal cell death [12]. ALDH2 exerts a major role in aldehyde detoxification in mitochondria, and attenuates or ablates neuronal mitochondrial damage. Reactive aldehydes, including MDA, 4-HNE and 1-palmitoyl-2-oxovaleroyl phosphatidyl choline (POVPC), all of which are potential substrates for ALDH2, are elevated in ischemic stroke injury [1, 12]. Higher levels of 4-HNE and MDA were found in the serum of strokeprone hypertensive rats compared with normotensive WKY rats [6]. Interestingly, only 4-HNE, but not MDA, was elevated in stroke-prone hypertensive rats compared with hypertensive rats, suggesting a role of 4-HNE as a possible biomarker for stroke. 


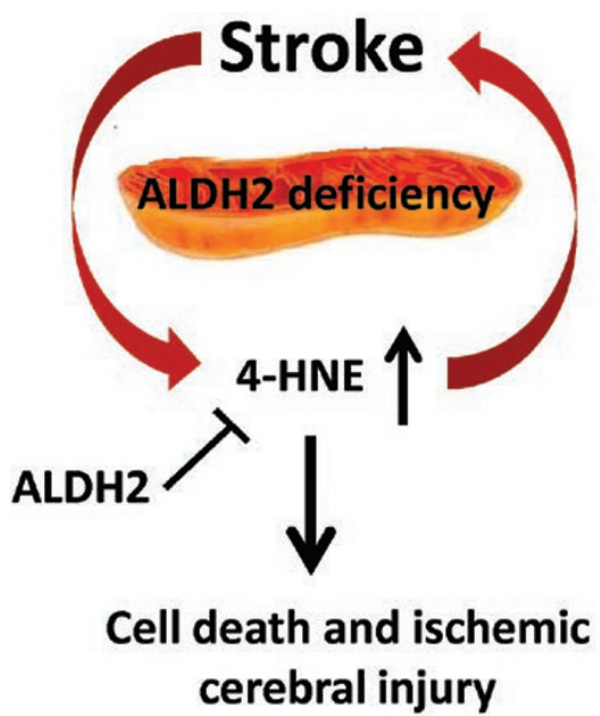

Figure 1 Schematic diagram depicting the possible interplay between ischemic stroke and elevated serum 4-HNE levels. Serum 4-HNE levels positively correlates with stroke injury and remains elevated after stroke. Deficiency and activation of ALDH2 significantly accentuates and attenuates stroke-associated cerebral ischemia injury, respectively.

Given that approximately $40 \%$ of the East Asian population carries an ALDH2*2 mutant allele with dramatic reduction in ALDH2 enzymatic activity, the current observation suggest that $\mathrm{ALDH} 2$ mutation serves as a risk factor for stroke [6]. Unlike its reported role in the heart, little information is available for ALDH2 in the brain and cerebrovascular function. Like all animal studies, caution needs to be taken to translate experimental findings to a clinical setting. It is noteworthy that the animal outcome studies were performed at a relatively short period after stroke. A longer time window should be essential to the ultimate assessment of stroke injury. Further studies are needed to uncover the precise mechanism behind the regulation of ALDH2 in stroke.
Aijun Sun ${ }^{1,2}$, Jun Ren ${ }^{1,2}$

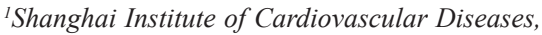
Zhongshan Hospital, Fudan University, Shanghai 200032, China; ${ }^{2}$ Center for Cardiovascular Research and Alternative Medicine, University of Wyoming, Laramie, WY 82071, USA

Correspondence: Jun Ren

Tel: 1-307-766-6131; Fax: 1-307-766-2953

E-mail: jren@uwyo.edu

\section{References}

1 Traylor M, Farrall M, Holliday EG, et al. Lancet Neurol 2012; 11:951-962.

2 Bevan S, Traylor M, Adib-Samii P, et al. Stroke 2012; 43:3161-3167.

3 Manolio TA, Collins FS, Cox NJ, et al. $\mathrm{Na}$ ture 2009; 461:747-753.

4 Gretarsdottir S, Thorleifsson G, Manolescu A, et al. Ann Neurol 2008; 64:402-409.

5 Gudbjartsson DF, Holm H, Gretarsdottir S, et al. Nat Genet 2009; 41:876-878.

6 Guo JM, Liu AJ, Zang P, et al. Cell Res 2013; 23:915-930.

7 Peng GS, Yin SJ. Hum Genomics 2009; 3:121-127.

8 Kato N, Takeuchi F, Tabara Y, et al. Nat Genet 2011; 43:531-538.

9 Doser TA, Turdi S, Thomas DP, et al. Circulation 2009; 119:1941-1949.

10 Koda K, Salazar-Rodriguez M, Corti F, et al. Circulation 2010; 122:771-781.

11 Ma H, Li J, Gao F, et al. J Am Coll Cardiol 2009; 54:2187-2196.

12 Osman MM, Lulic D, Glover L, et al. Neuropeptides 2011; 45:359-368. 